\title{
Comparison between visual and laser- fluorescence diagnosis of occlusal surfaces of first permanent molars. Literature review and clinical cases.
}

\section{Lilyana Shtereva ${ }^{1}$, Veselina Kondeva ${ }^{2}$}

1. Assistant professor in the Department of Pediatric Dentistry, Faculty of Dental Medicine, Medical University - Plovdiv, Plovdiv, Bulgaria;

2. Associate professor in the Department of Pediatric Dentistry, Faculty of Dental Medicine, Medical University - Plovdiv, Plovdiv, Bulgaria;

\begin{abstract}
Introduction: Proper examination of occlusal surfaces is one of the main objects of scientific interest in dental medicine. A number of diagnostic methods have been established, which purpose is more accurate diagnostics, however, the accurate diagnosis of the occlusal surface of newly erupted first permanent molars is still a challenge for dental specialists.
\end{abstract}

Aim: The aim of this study is to provide information about the application of visual and laser-fluorescence methods for diagnosis of occlusal surfaces of newly erupted first permanent molars and to present personal clinical cases. 
Materials and methods: Articles in Bulgarian and English language were selected and read in full. We have also conducted our own studies on the application of visual diagnosis and laser-fluorescence diagnosis with Vistacam. 46 newly erupted first permanent molars of 12 children from 5 to 8 years old are monitored.

Results: The results of the literature studies confirm Vistacam as a reliable auxiliary diagnostic method, a useful complement to visual diagnostics. Our studies confirm that there is a correlation between the results reported of a diagnosis with Vistacam and visual examination using ICDAS II system. The ICDAS II system used in visual diagnostics is reliable enough, and the application of laser-fluorescence confirms and visualizes the presence or absence of changes in the mineralization of hard dental tissues.

Conclusion: The data obtained from literature sources as well as from our own clinical cases will serve as a basis for future studies connected with assessment of occlusal surfaces of newly-erupted first permanent molars before and after silanization, previously diagnosed as sound using both methods.

Keywords: first permanent molars, occlusal surfaces sealants, laser-fluorescence, visual diagnosis

\section{Introduction}

Proper examination of the occlusal surface is one of the main objects of scientific interest in dental medicine. A number of diagnostic methods and tools have been created, the main purpose of which is more accurate diagnostics. At the moment, a wide range of methods and tools are being used as a result of the technological development. The ideal diagnostic method should be easy to apply, non-invasive, reproducible, sensitive. In addition, it should be equally well received by patients and dental practitioners and enable early diagnosis in both -practice and in research. It should provide information that could help our decisions for prevention and treatment in order to assure patient's long-term oral health. Such an universal method has not been discovered yet, but the reliability of the results can be significantly improved by a combination of diagnostic methods and diagnostic tools.

\section{Aim}

The aim of this study is to provide information about the application of visual and laser-fluorescence methods for diagnosis of occlusal surfaces of newly erupted first permanent molars and to present personal clinical cases. And to compare the effectiveness of diagnostic methods - visual diagnosis using the ICDAS system and laser fluorescence diagnosis with Vistacam.

\section{Materials and methods}

Articles in Bulgarian and English language were selected and read in full. We have also conducted our own studies on the application of visual diagnosis using the ICDAS II system (table 1) and laser-fluorescence 
diagnosis with Vistacam (fig.1). 46 newly erupted first permanent molars of 12 children from 5 to 8 years old are monitored.

Table.1. ICDAS II codes

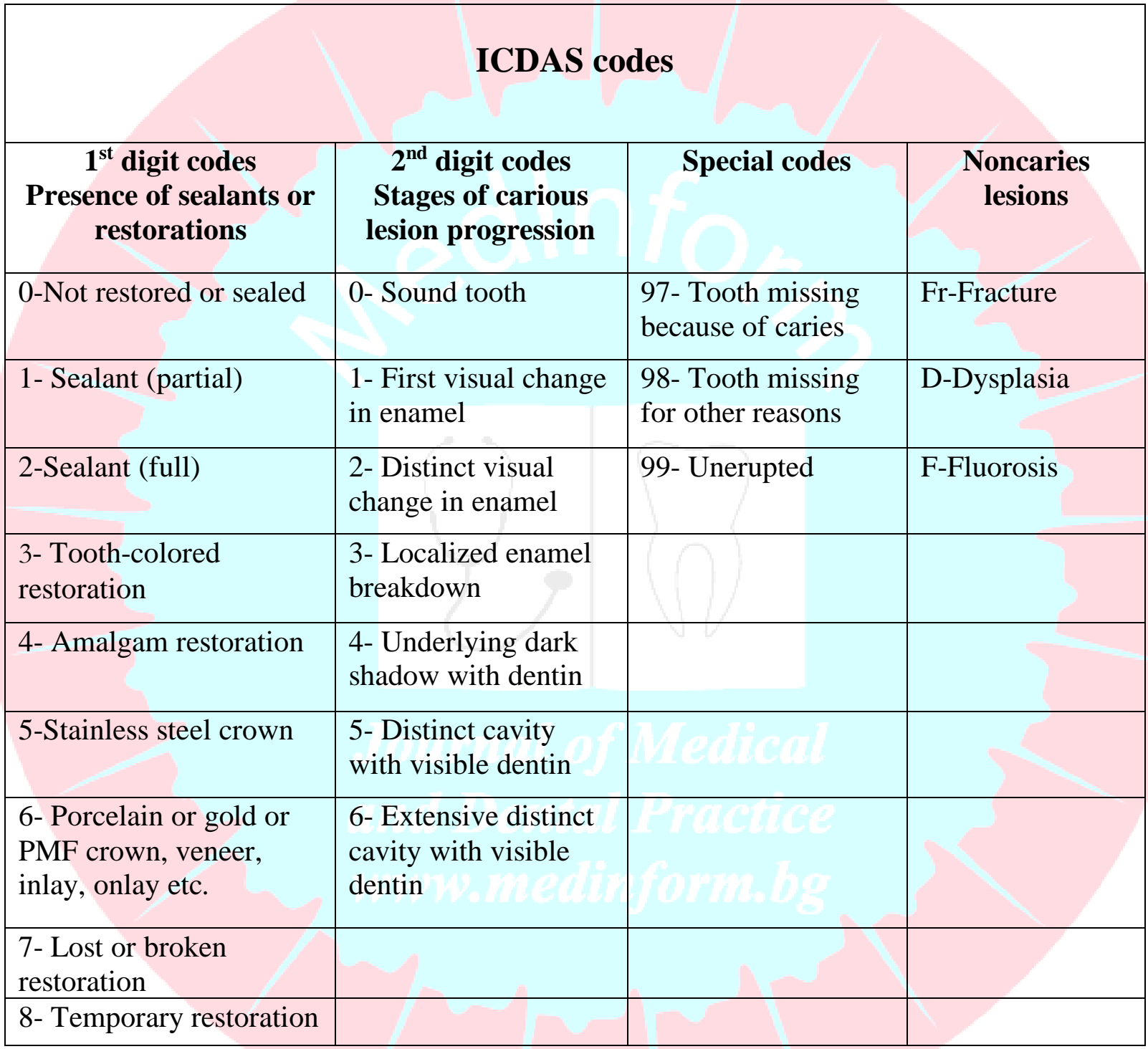




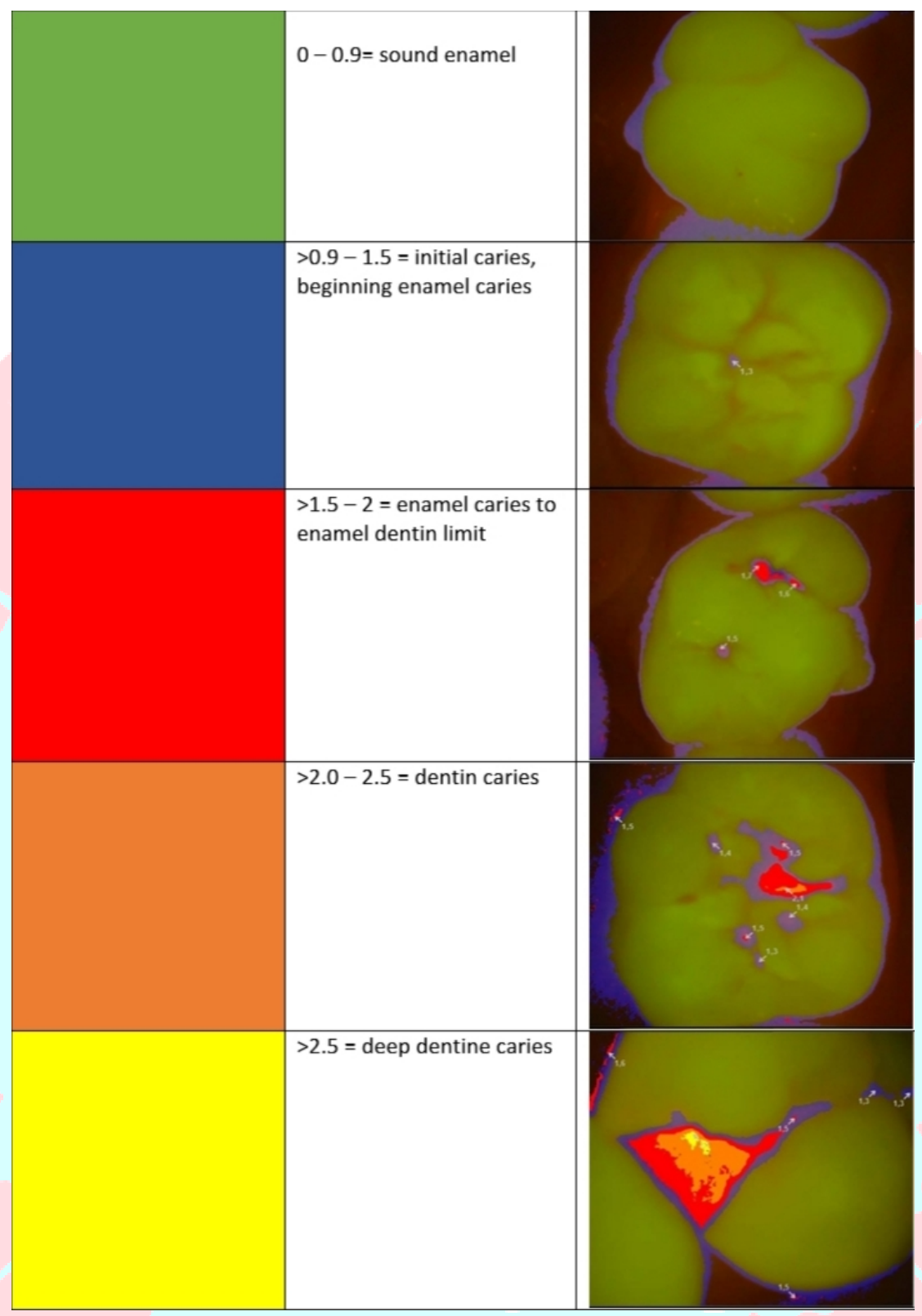

Fig.1. Vistacam codes

\section{Results}

\section{Review of the literature. Visual diagnostics}

Visual inspection of macroscopically intact occlusal surfaces is a method with limited sensitivity $(1,2)$. By increasing the experience and training of examiners, as well as by the use of more precise criteria for visual 
diagnosis, the accuracy of the diagnosis and the sensitivity of the method are increased. In recent years, the need to use a probe in the assessment of occlusal dental surfaces has been debated. The role of the dental probe for diagnosis of an already cavitated caries is indisputable, however most of the authors are convinced that probing has no place in the modern management of initial dental caries, when uncavitated carious lesions are treated by remineralization, sealants, minimally invasive techniques and microobturations $(1,3,4)$. Visual observation is carried out after drying the surface and using direct light. The main advantage of the visual inspection is the advantage of not damaging the enamel. Trough visual assessment the color of the fissure is monitored. However, the presence of a change in the color of the fissure is not always an absolute indication of intervention (1,5). It can be due to both colored carious dentin or retention of pigments from nutritional degradation. Difficulties in diagnosing occlusal caries through visual inspection have led to the creation of an International Caries Detection and Assessment System (ICDAS), and later the development of ICDAS II $(6,7)$. This system makes it possible to take into account even the earliest changes in the enamel, as well as the presence of dental caries. ICDAS II shows a satisfactory correlation between the reported changes in the enamel and dentin assessed using the ICDAS II system, compared with histological measurements made.

\section{Laser-fluorescence diagnostics}

Fluorescence is the ability of some materials and tissues to absorb light of a given wavelength and to emit light of varying wavelengths. The phenomenon of fluorescence of hard dental tissues was first described in 1911. One of the applications of fluorescence is to diagnose dental caries.

The Vista Cam ix (Durr Dental, Bietigheim- Bissingen, Germany) intraoral self-calibrating fluorescence camera is a diagnostic tool for quantitative assessment of dental caries with a high sensitivity for demineralized lesions detection in enamel and dentin. The Vista Cam iX LEDs emits high-energy blue-violet light at $405 \mathrm{~nm}$ on the occlusal tooth area. At this wavelength porphyrins produced by caries-related bacteria, emit red light, containing less energy, in contrast to sound enamel, characterized by green light $(8,9,10,11)$.

Carious tissue and healthy tissue emit fluorescence at different intensities when excited by light at specific wavelengths. The fluorescence is recorded by the camera, transferred and processed by a software (DBSWIN, Durr) and then stored. As result a digital image show lesions in different colours shades with numerical score between 0 and 3, predicting the extent and depth of carious demineralization. The device is easy to handle in general practice and offer a non- invasive tool for occlusal caries detection $(9,10,11,12)$.

A study conducted in Rome and published in 2016 compares the effectiveness of ICDAS visual diagnostics and laser-fluorescence diagnostics with VistaCam iX-oral camera for the early diagnosis and evaluation of permanent tooth caries. 13 to 30 years 10 males and 16 females, 200 permanent teeth (53 premolars and 147 molars) were examined. The conclusion was that there is a correlation between the results reported with ICDAS II and VistaCam iX Proof. The intraoral camera is a useful additional method to the visual diagnosis with ICDAS II (13).

Similar results are found in a similar study conducted in 2017, which found a high level of correspondence between the sensitivity and specificity of the two methods of diagnosis: values for Vistacam 0.89 / 0.96 and values for ICDAS 0.84 / 0.99 (9). 


\section{Literature review and clinical cases}

Our own studies on the visual diagnostics of occlusal surfaces in newly erupted first permanent molars show a correlation between the results reported with the help of ICDAS II system and VistaCam. The data obtained from the visual examination using the ICDAS II system are accurate, but the visualization of the available or missing changes on the occlusal surface makes it possible to both objectify the results and track them over time. A major advantage of VistaCam laser fluorescence is the ability to evaluate the depth of a carious lesion, which greatly helps taking the right clinical decision.

The presented clinical cases (Figure 2, Figure 3) illustrate the correspondence between the two diagnostic methods. The stained areas of the occlusal surfaces observed during visual diagnostics are reported to be affected to varying degrees when performing fluorescence diagnostics with Vistacam. The image processed by the software of the program gives color and digital information about the depth of the lesions, which in the presented cases are limited to the enamel.

The first case presented is D.K., 7 years old (Fig. 2), after visual diagnostics, the visible changes in the enamel of the examined occlusal surface were corresponding to the digital coding: code 03 was given using the ICDAS II system, corresponding to the image obtained after conducting laser fluorescence diagnostics with Vistacam, where the affected areas were depicted in colors (other than green color- characteristic for sound tooth structures) and numbers. Part of the pits and fissures are colored blue and digital coding 1.21.3- indicating the presence of initial caries in the enamel, and some areas are colored red and have a digital coding-1.6-corresponding to the presence of caries in the enamel, which doesn't pass the dentino-enamel junction.
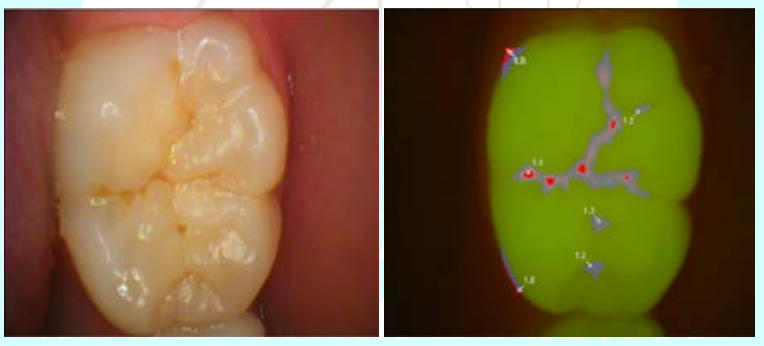

Fig. 2 D.K., 7 years old, first permanent mandibular molar: Clinical case 1

The second clinical case presented: V.A., 8 years old (Fig. 3), after visual diagnostics, visible changes in the enamel of the examined occlusal surface were corresponding to the digital coding: code 03 according to the ICDAS II system, corresponds to the image obtained after diagnostics by laser fluorescence with Vistacam, depicting the affected areas in colors and numbers. Part of the pits and fissures are colored blue and the digital coding is 1.3-1.4- which shows presence of initial caries in the enamel, and some areas are red and have a digital coding-1.7-corresponding to presence of caries in the enamel, not passing the dentino-enamed junction. 

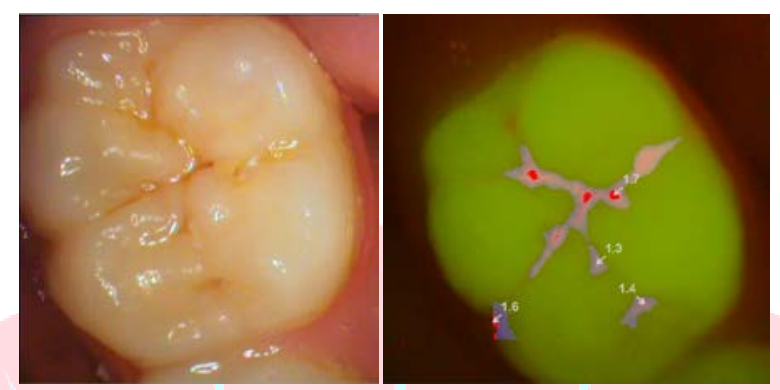

Fig.3 V.A., 8 years old, first permanent mandibular molar, Clinical case 2

\section{Discussion}

The results after reading and analyzing the articles which met the inclusion criteria show that Vistacam is confirmed to be a reliable but additional diagnostic method, a useful complement to visual diagnosis with ICDAS II system. The intraoral camera enables long-term follow-up of the lesions and improves visual inspection in order to minimize errors during diagnosis, enables long-term follow-up of occlusal surfaces and detection of changes over time (9). Along with these advantages, laser fluorescence diagnostics with Vistacam has its disadvantages as well. Survey conducted in Brazil in 2017 concludes that the chance of false-positive results conducted by visual dianosis using the ICDAS system is lower than that of fluorescence diagnosis with Vistacam, which may lead to overdiagnosis and the inappropriate treatment plan (9).

However, a major advantage of Vistacam is that the device does not require pre-calibration, and can be used by all clinicians. Our studies confirm that there is a correlation between the results acquired with visual diagnosis using the ICDAS II system and laser-fluorescent diagnostics with Vistacam, and that the intraoral fluorescence camera can qualitatively assist visual inspection with the ICDAS II system. The accurate diagnosis of the presence or absence of occlusal caries through only visual method is a challenge for the dentist when there is an absence of cavitation.

Other reasons limiting proper diagnostics are the use of inappropriate probes and mirrors; improper use of diagnostic probes whereby part of the enamel is broken; lack of drying, whereby saliva disguises the presence of dental caries.

In recent years, a series of studies have been published proving that the use of an intraoral camera or the use of fluorescence diagnostic methods in dental practice significantly improves the diagnosis of occlusal caries $(14,15)$.

VistaCam intraoral camera enables long-term follow-up of lesion remineralization and enhances visual inspection to minimize errors during diagnosis.

Based on the information received, the level of objectivity in ICDAS II diagnostics is expected to have a level of objectivity similar to, or just more than the one obtained by using VistaCam. The enlarged image obtained with the help of Vistacam intraoral camera can add essential information needed for visual diagnostics, 
further facilitating the diagnosis of dental caries, or defects in the obturations or sealants of already obturated or sealanized tooth surfaces. The enlarged image also allows more accurate treatment or planning prophylactic measures.

\section{Conclusion}

The data obtained from literature sources as well as from our own studies will be taken into consideration and serve as a basis for comparison of both diagnostic methods: initial inspection and follow-up of occlusal surfaces of newly-erupted first permanent molars before and after sealanization, with previously diagnosed sound occlusal surfaces using both methods- visual inspection and laser-fluorescence.

\section{References}

1. Кондева В., М.Куклева Оклузален кариес при постоянни детски зъби, ЕТ “Васил Петров”, 2010; 41-3.

2. Lussi A. Comparison of different methods for the diagnosis of fissure caries without cavitation. Caries Res 1993; 27: 409-416.

3. Axelsson P. Diagnosis and risk prediction of dental caries. Quintessence Publishing Co, Inc 2000.

4. Kühnisch J, K. Bücher, Hickel. The intra/inter-examiner reproducibility of the new DIAGNOdent Pen on occlusal sites. J Dent 2007; 35(6): 509-512.

5. Ferreira-Zandoná AG, M. Anloui, B. Beiswander, R. et al. An in vitro comparison between laser fluorescence and visual examination for detection of demineralization in occlusal pits and fissures. Caries Res 1998; 32(3): 210-219.

6. Jablonski-Momeni A. , V. Stachniss, D. N. Ricketts et al, Reproducibility and accuracy of the ICDAS-II for detection of occlusal caries in vitro, Caries Research, vol. 42, no. 2, pp. 79-87, 2008.

7. Jablonski-Momeni A., J. Stucke, T. Steinberg et al., Use of ICDAS-II, Fluorescence-Based Methods, and Radiography in Detection and Treatment Decision of Occlusal Caries Lesions: An In Vitro Study, International Journal of Dentistry, 2012, Article ID 371595, doi:10.1155/2012/371595

8. Lussi A, Megert B, Longbottom C, et al. Clinical performance of a laser fluorescence device for detection of occlusal carieslesions. . Eur J Oral Sci. 2001 Feb;109:149.

9. Presoto, Trevisan, Andrade et al. "Clinical effectiveness of fluorescence, digital images and ICDAS for detecting occlusal caries "; Rev Odontol UNESP. 2017 Mar-Apr; 46(2): 109-115

10. Jablonski-Momeni A., M. Heinzel-Gutenbrunner, S. Klein. In vivo performance of the VistaProof fluorescence-based camera for detection of occlusal lesions. Clin Oral Investig. 2014; 18(7):1757-62.

11. Jablonski-Momeni A, M. Heinzel-Gutenbrunner, G. Vill. Use of a fluorescence-based camera for monitoring occlusal surfaces of primary and permanent teeth. Int J Paediatr Dent. 2016 ;26(6):448-56.

12. De Benedetto MS, C. Morais, T. Novaes et al. Comparing the reliability of a new fluorescence camera with conventional laser fluorescence devices in detecting caries lesions in occlusal and smooth surfaces of primary teeth. Lasers Med Sci. 2011 ;26(2):157-62 
13. Guerra F., D. Corridore,M. Mazur et al. Early caries detection: comparison of two procedures. A pilot study. Senses Sci 2016; 3 (4):317-322

14. Erten H, M.Uctasli, Z. Akarslan et al;. The assessment of unaided visual examination, intraoral camera and operating microscope for the detection of caries lesions. Oper Dent 2005; 30(2): 190-194.

15. Jones RS, C. Darling, J. Featherstone, D. Fried. Imaging artificial caries on the occlusal surfaces with polarization-sensitive optical coherence topography. Caries Res 2006; 40(2): 8189.

\section{Corresponding author:}

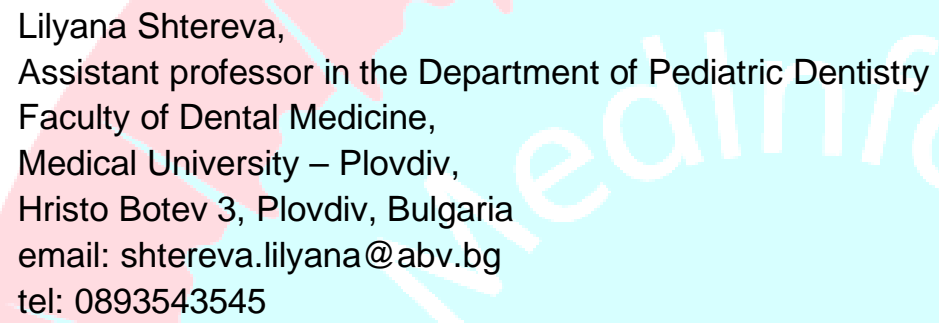

